\title{
Divergent Hypertension-Inducing Manifestations between Anxiety and Depression: A Cardio (Anxiety) Vascular (Depression) Perspective
}

\author{
Firulescu L ${ }^{1,2}$, May RW', Ferrer GF' ${ }^{2}$, Fincham FD $^{3}$ \\ and Sanchez-Gonzalez MA ${ }^{1,2 *}$ \\ ${ }^{1}$ Division of Clinical \&Translational Research, Larkin \\ Community Hospital, USA \\ ${ }^{2}$ Department of Psychiatry, Larkin Community Hospital, \\ USA \\ ${ }^{3}$ Family Institute, Florida State University, USA \\ *Corresponding author: Marcos A. Sanchez-Gonzalez, \\ Division of Clinical \& Translational Research Larkin \\ Community Hospital, 7000 SW 62nd Ave. South Miami, \\ FL 33143, USA
}

Received: October 17, 2017; Accepted: October 24, 2017; Published: October 31, 2017

\section{Perspective}

High blood pressure, an important risk factor for developing cardiovascular disease, seems to be more prevalent in patients with comorbid psychopathology [1]. Among all psychiatric disorders, anxiety has the highest lifetime prevalence, and tends to be comorbid with major depressive disorder. A predictor of cardiovascular morbidity and mortality, anxiety exacerbates and accelerates the progression of coronary artery disease, arrhythmias, stroke and hypertension [2]. Similarly, Major Depressive Disorder (MDD), a major cause of disability worldwide, is associated with the development of adverse cardiovascular outcomes, including hypertension, arrhythmias, increased left ventricular mass, and myocardial infarction [3]. Prior research has shown that MDD and anxiety are independent risk factors for hypertension [4]. Whether via a shared mechanism or different pathways, the exact mechanism by which hypertension develops in patients with depression and anxiety is poorly understood. The aim of the present document is to propose a model of hypertension development using the cardio (anxiety) vascular (depression) concept of pathogenesis. The model proposes that while depression has a strong impact on the sympathetically dominated organs (e.g. vasculature), anxiety promotes vagal withdraw and a more cardiac driven increase in blood pressure.

Several studies have shown a robust association between vascular dysfunction and MDD. Moreover, our group has documented that depressive symptoms are associated with increased sympathetic vasomotor tone, and to some extent, an early manifestation of dysautonomia [5]. Dysautonomia, including increased Sympathetic Nervous System (SNS) activity and/or decreased vagal tone, may be a common pivotal factor between depression and cardiovascular diseases [6]. In laboratory measurements, Seldenrijk et al. [7] reported that depression severity is associated with lower carotid compliance. We demonstrated that higher depressive symptoms, in individuals without major depressive disorder, are associated with cardiac hyperactivity during SNS stimulation, contributing to increased aortic hemodynamics, increased pressure wave reflection and ventricular work. Light et al. [8] showed that catecholamine concentrations were associated with exaggerated Blood Pressure (BP) responses to laboratory stressors in female subjects with depressive symptoms. Similarly home-based measurements, by means of ambulatory BP, have shown that morning Systolic BP (SBP) surge and nocturnal SBP dipping are positively and negatively correlated with depressive symptoms, respectively [9]. Furthermore, the Ambulatory Arterial Stiffness Index (AASI) may be increased in patients with major depressive disorder, owing to increased SNS activity thus impacting the arterial tree [10]. Together these studies suggest that depression has a profound impact on the vasculature, most likely to be mediated by increased SNS tone, and hence markers of neurovascular modulation may better demonstrate the association between depression and high BP [5].

On the same note, vascular autonomic modulation is known to have a predominant SNS tone [11], hence the evaluation of the rhythmical oscillations in SBP in the Low-Frequency Domain (LFSBP), a surrogate of sympathetic vasomotor tone [12], may be appropriate for understanding the influence of SNS hyperactivity on vascular function in MDD. Research demonstrated that a blunted LFSBP response during a stress test was associated with higher depressive symptoms, which may be indicative of dysautonomia. Data demonstrated that depressive symptoms were associated with dysautonomia and increased sympathetic vasomotor tone, even in the absence of clinically meaningful cardiovascular alterations, suggesting that LFSBP could be an early indicator of increased cardiovascular risk in those with increased depressive symptom scores [5].

In contrast, studies have shown that anxiety may have adverse effects on cardiac autonomic modulation by decreasing vagal tone, increasing sympathetic activity and diminishing cardiac reactivity to stress [13]. On the other hand, attenuated cardiac response to stress in people with high anxiety symptoms is likely driven by increased vagal withdraw, as evidenced by the blunted high frequency of heart rate variability (HF) response during a stress test [14]. Similarly, Shinba et al. [15] reported a negative correlation between anxiety scores, heart rate and heart rate variability in response to a stress task. In addition, Yu et al. [16] reported that anxiety was associated with betaadrenergic receptor desensitization, underlining that anxiety may attenuate cardiovascular responses to stress. Together these studies seem to suggest that anxiety has a more pronounced anticholinergic effect manifested as increased heart rates.

Although the anxiety induced cardiovagal withdraw effects are more pronounced than those of depression, the comorbid nature of these psychiatric symptoms could potentially instigate a common
Ann Depress Anxiety - Volume 4 Issue 2 - 2017

ISSN : 2381-8883 | www.austinpublishing group.com

Sanchez-Gonzalez et al. @ All rights are reserved
Citation: Firulescu L, May RW, Ferrer GF, Fincham FD and Sanchez-Gonzalez MA. Divergent HypertensionInducing Manifestations between Anxiety and Depression: A Cardio (Anxiety) Vascular (Depression) Perspective. Ann Depress Anxiety. 2017; 4(2): 1087. 
Table 1: Summarizes the impact of anxiety and depression on selected cardiovascular physiological parameters.

\begin{tabular}{|c|c|c|c|}
\hline $\begin{array}{l}\text { Cardiovascular } \\
\text { Parameter }\end{array}$ & Technical Approach & Anxiety & Depression \\
\hline $\mathrm{HR}(\mathrm{bpm})$ & ECG & $\uparrow \uparrow$ & $=$ \\
\hline MAP $(\mathrm{mmHg})$ & Brachial Sphygmomanometer & $\uparrow$ & $\uparrow \uparrow$ \\
\hline RPP (bpm X mmHg) & ECG/Sphygmomanometer & $\uparrow$ & $\uparrow$ \\
\hline HR-24h (bpm) & \multirow{4}{*}{ Ambulatory Monitoring (Spacelabs) } & $\uparrow$ & $=$ \\
\hline MAP-24h (mmHg) & & $=$ & $\uparrow$ \\
\hline RPP-24h (bpm X mmHg) & & $=$ & $=$ \\
\hline AASI & & $=$ & $\uparrow$ \\
\hline $\mathrm{BRS}(\mathrm{ms} / \mathrm{mmHg})$ & \multirow{2}{*}{ Finger plethysmography } & $\downarrow$ & $\downarrow \downarrow$ \\
\hline $\mathrm{BRD}(\mathrm{sec})$ & & $\uparrow$ & $\uparrow \uparrow$ \\
\hline HRV & \multirow{2}{*}{ Power spectral analysis heart rate variability } & $\downarrow \downarrow$ & $=\downarrow$ \\
\hline nLF & & $\uparrow \uparrow$ & $\uparrow$ \\
\hline LFSBP $\left(m m \mathrm{mg}^{2}\right)$ & Power spectral analysis blood pressure variability & $=$ & $\uparrow \uparrow$ \\
\hline Alx (\%) & \multirow{5}{*}{ Applanation tonometry } & $=$ & $\uparrow \uparrow$ \\
\hline Aortic MAP (mmHg) & & $=$ & $\uparrow$ \\
\hline $\mathrm{STI}(\mathrm{mm} \mathrm{Hg} / \mathrm{s} . \mathrm{min}-1)$ & & $\uparrow \uparrow$ & $\uparrow$ \\
\hline DTI (mm Hg/s.min-1) & & $\uparrow$ & $\uparrow \uparrow$ \\
\hline SEVR (\%) & & $\downarrow \downarrow$ & $=$ \\
\hline
\end{tabular}

HR: Heart Rate; MAP: Mean Arterial Pressure; RPP: Rate Pressure Product; HR-24h: Ambulatory 24 hour mean heart rate; MAP-24h: Ambulatory 24 Hour Mean Arterial Pressure; P: Pressure; RPP-24h: ambulatory rate pressure product; BRS: Baroreflex Sensitivity; BRD: Baroreflex Delay; HRV: Heart Rate Variability; nLF: Normalized Low Frequency Component of HRV; AASI: Ambulatory Arterial Stiffness Index; LFSBP: Low Frequency Component of Systolic Blood Pressure Variability; Aix: Augmentation Index; STI: Systolic Time Integral; DTI: Diastolic Time Integral; SEVR: Subendocardial Viability Ratio

hypertension inducing pathophysiological mechanism? Some studies suggest that the hemodynamic and vascular responses (low frequency component of systolic blood pressure variability, LFSBP; stiffness index, $\mathrm{SI}_{\mathrm{DVP}}$ ) to stress are amplified in individuals with high anxiety, a reaction opposite to the HRV attenuation. These results demonstrate that individuals with high anxiety display higher resting LFSBP levels than those with low anxiety. Significant differences were found between groups with high and low anxiety scores in Baroreflex Sensitivity (BRS) and Baroreflex Delay (BRD), suggesting impaired baroreflex function associated with anxiety. The relationship between indices of autonomic modulation and anxiety seems to be mediated through increased sympathetic activity and reduced cardiovagal control probably due to a reduced baroreflex function [14] both of which are usually found in MDD.

Altogether, prior studies as well as our research have documented that depression scores are significantly associated with higher brachial systolic blood pressure, brachial diastolic blood pressure, brachial mean blood pressure, aortic systolic blood pressure, aortic diastolic blood pressure, aortic mean blood pressure, aortic pulse pressure, and Diastolic pressure-Time Index (DTI). In comparison, anxiety scores are associated with higher heart rate, systolic blood pressure, brachial pulse pressure, aortic pulse pressure, Systolic pressure-Time Index (STI), DTI, and lower subendocardial viability index (SVI; surrogate of coronary perfusion). Overall, depression scores are significantly associated with a greater increase in all cardiovascular parameters except for SVI where there is significant decrease; anxiety scores with a greater increase in heart rate, but a decrease in brachial diastolic blood pressure, brachial mean blood pressure, aortic systolic blood pressure, aortic diastolic blood pressure, aortic mean blood pressure,
DTI, and SVI (Table 1) [17].

In sum, depression and anxiety are known contributors to the development of hypertension. It appears that anxiety induces vagal withdraw while depression seems to be associated with increased SNS predominance, thus affecting the organs dominated by each of the autonomic nervous system branches. That is to say a cardio (anxiety) - vascular (depression) model of the development of hypertension in psychopathology. Here we propose that methodological prospective approaches (Table 1) in such areas must consider the autonomic dominance of the organ studied to access the differential anxiety (parasympatholytic) and depression (adrenergic) effects in the pathogenesis of hypertension, and ultimately more targeted therapeutic interventions.

\section{References}

1. Almas A, Patel J, Ghori U, Ali A, Edhi Al, Khan MA. Depression is linked to uncontrolled hypertension: a case-control study from Karachi, Pakistan. J Ment Health. 2014; 23: 292-296.

2. Kawachi I, Sparrow D, Vokonas PS, Weiss ST. Symptoms of anxiety and risk of coronary heart disease. The Normative Aging Study. Circulation. 1994; 90: $2225-2229$

3. Sanchez-Gonzalez MA, May RW, Brown PC, Koutnik AP, Fincham FD. Depressive symptoms contribute to increased wave reflection during cold pressor test in young adult men. Am J Hypertens. 2013; 26: 778-783.

4. Sunbul M, Sunbul EA, Kosker SD, Durmus E, Kivrak T, lleri C, et al. Depression and anxiety are associated with abnormal nocturnal blood pressure fall in hypertensive patients. Clin Exp Hypertens. 2014; 36: 354-358.

5. Sanchez-Gonzalez MA, May RW, Koutnik AP, Kabbaj M, Fincham FD. Sympathetic vasomotor tone is associated with depressive symptoms in young females: a potential link between depression and cardiovascular disease. Am J Hypertens. 2013; 26: 1389-1397. 
6. Hamer M, Malan L. Sympathetic nervous activity, depressive symptoms, and metabolic syndrome in black Africans: the sympathetic activity and ambulatory blood pressure in Africans study. Stress. 2012; 15: 562-568.

7. Seldenrijk A, Van Hout HP, Van Marwijk HW, de Groot E, Gort J, Rustemeije C, et al. Depression, anxiety, and arterial stiffness. Biol Psychiatry. 2011; 69: 795-803.

8. Light KC, Kothandapani RV, Allen MT. Enhanced cardiovascular and catecholamine responses in women with depressive symptoms. Int J Psychophysiol. 1998; 28: 157-166.

9. FitzGerald L, Ottaviani C, Goldstein IB, Shapiro D. Effects of dipping and psychological traits on morning surge in blood pressure in healthy people. $J$ Hum Hypertens. 2012; 26: 228-235.

10. Li Y, Wang JG, Dolan E, Gao PJ, Guo HF, Nawrot T, et al. Ambulatory arteria stiffness index derived from 24-hour ambulatory blood pressure monitoring. Hypertension. 2006; 47: 359-364.

11. Julien C, Malpas SC, Stauss HM. Sympathetic modulation of blood pressure variability. J Hypertens. 2001; 19: 1707-1712.

12. Malliani A, Pagani M, Lombardi F, Cerutti S. Cardiovascular neural regulation explored in the frequency domain. Circulation. 1991; 84: 482-492.
13. Mankus AM, Aldao A, Kerns C, Mayville EW, Mennin DS. Mindfulness and heart rate variability in individuals with high and low generalized anxiety symptoms. Behav Res Ther. 2013; 51: 386-391.

14. Sanchez-Gonzalez MA, Guzik P, May RW, Koutnik AP, Hughes R, Muniz $\mathrm{S}$. Trait anxiety mimics age-related cardiovascular autonomic modulation in young adults. J Hum Hypertens. 2015; 29: 274-280.

15. Shinba T, Kariya N, Matsui Y, Ozawa N, Matsuda Y, Yamamoto K. Decrease in heart rate variability response to task is related to anxiety and depressiveness in normal subjects. Psychiatry Clin Neurosci. 2008; 62: 603-609.

16. Yu BH, Kang EH, Ziegler MG, Mills PJ, Dimsdale JE. Mood states, sympathetic activity, and in vivo beta-adrenergic receptor function in a normal population. Depress Anxiety. 2008; 25: 559-564.

17. Sanchez-Gonzalez MA, May RW, Koutnik AP, Fincham FD. Impact of negative affectivity and trait forgiveness on aortic blood pressure and coronary circulation. Psychophysiology. 2015; 52: 296-303.
Ann Depress Anxiety - Volume 4 Issue 2 - 2017

ISSN : 2381-8883 | www.austinpublishing group.co

Sanchez-Gonzalez et al. () All rights are reserved
Citation: Firulescu L, May RW, Ferrer GF, Fincham FD and Sanchez-Gonzalez MA. Divergent HypertensionInducing Manifestations between Anxiety and Depression: A Cardio (Anxiety) Vascular (Depression) Perspective. Ann Depress Anxiety. 2017; 4(2): 1087. 